\title{
Inhibition of Maintenance DNA Methylation by Stella
}

Soichiro Funaki ${ }^{1,2,3}$, Toshinobu Nakamura ${ }^{3,4,5,6}$, Tsunetoshi Nakatani ${ }^{1,3}$, Hiroki Umehara $^{3,7}$, Hiroyuki Nakashima ${ }^{7}$, Toru Nakano ${ }^{1,5,6,7}$

${ }^{1}$ Department of Pathology, ${ }^{2}$ Department of General Thoracic Surgery, Graduate School of Medicine, ${ }^{7}$ Graduate School of Frontier Biosciences, Osaka University, Osaka, 565-0871, Japan

${ }^{4}$ Nagahama Institute of Bio-Science and Technology, Shiga, 526-0829, Japan

${ }^{6}$ JST, CREST, Saitama, 332-0012, Japan

${ }^{3}$ These authors contributed equally to this work.

${ }^{5}$ Correspondence should be addressed to

T. Nakamura (e-mail: tnakamura@nagahama-i-bio.ac.jp) or T. Nakano (e-mail: tnakano@patho.med.osaka-u.ac.jp)

Keywords: Stella; Np95; Dnmt1; DNA demethylation 


\section{Abstract}

DNA methylation is a key epigenetic regulator in mammals, and the dynamic balance between methylation and demethylation impacts various processes, from development to disease. DNA methylation is erased during replication when DNA methyltransferase 1 (DNMT1) fails to methylate the daughter strand, in a process known as passive DNA demethylation. We found that the enforced expression of Stella (also known as PGC7, Dppa3), a maternal factor required for the maintenance of DNA methylation in early embryos, induced global DNA demethylation in NIH3T3 cells. This demethylation was caused by the binding of Stella to Np95 (also known as Uhrf1, ICBP90) and the subsequent inhibition of DNMT1 recruitment. Considering that impaired DNA methylation profiles are associated with various developmental or disease phenomena, Stella may be a powerful tool with which to study the biological effects of global DNA hypomethylation. 


\section{Introduction}

Regulation of DNA methylation is essential for the control of gene expression in many biological processes, such as cell differentiation, development, and reprogramming [1,2]. Two DNA methylation processes are known: de novo DNA methylation and maintenance DNA methylation. In both cases, a methyl residue is added to the 5 position of the cytosine in CpG dinucleotides by DNA methyltransferases (DNMTs). De novo DNA methylation is the addition of a methyl residue to unmethylated DNA by so called de novo DNA methylases. In this case, the DNA methylation status cannot be maintained during DNA replication without the activity of the maintenance DNA methylase, DNMT1; however, DNA methylation is stably maintained if DNMT1 is appropriately recruited to the DNA replication fork [3].

Although DNMT1 is the catalytic enzyme involved in the methylation of newly synthesized hemi-methylated DNA, DNMT1 alone is not sufficient to maintain DNA methylation. Np95 (also known as Uhrf1 and ICBP90) is reported to be essential for the process as a result of its binding activity to hemi-methylated $\mathrm{CpG}$ dinucleotides through SET and RING finger-associated (SRA) domains [4,5]. Np95 tethers DNMT1 to hemi-methylated CpGs, which localize behind the replication fork, which is marked by the ring-shaped proliferating cell nuclear antigen (PCNA). 
We investigated the function of Stella, also known as PGC7 and Dppa3, an essential protein in early embryogenesis. Stella binds to the chromatin-containing dimethylated histone $\mathrm{H} 3$ lysine 9 (H3K9me2), which protects DNA methylation in zygotes [6]. To assess its molecular function in more detail, we expressed Stella in NIH3T3 cells. We expected that Stella would protect DNA methylation in somatic cells, as well as in early embryos; however, global DNA hypomethylation occurred via inhibition of the appropriate recruitment of DNMT1. We subsequently analyzed the molecular events underlying Stella-induced global DNA hypomethylation.

\section{Materials and Methods}

\section{Plasmids}

Stella was inserted into the EcoRI/SalI sites of the plasmid pMY-IRES-EGFP. Flag-tagged Np95, and its deletion mutants with stop codons, were inserted into the plasmid pcDNA4MycHisA.

\section{Cell culture}


NIH3T3 cells and Plat-E packaging cells [7] were maintained in Dulbecco's modified Eagle's Minimum Essential Medium, supplemented with $100 \mathrm{U} / \mathrm{mL}$ penicillin, streptomycin sulfate, and 10\% fetal bovine serum (FBS).

\section{Retroviral infection}

pMY-IRES-EGFP or pMY-Stella-IRES-EGFP retroviral vectors were introduced into Plat-E cells using FuGENE 6 transfection reagent (Roche), according to the manufacturer's recommendations. Cells were then incubated overnight at $37^{\circ} \mathrm{C}$, in the presence of 5\% CO2. Twenty-four hours after transduction, the medium was replaced. After an additional $24 \mathrm{~h}$, virus-containing supernatants derived from the Plat-E cultures were filtered through $0.45-\mu \mathrm{m}$ cellulose acetate filters and supplemented with $8 \mu \mathrm{g} / \mathrm{mL}$ polybrene. Target cells were incubated overnight with the virus/polybrene-containing supernatants. After infection of the cells, the medium was replaced with fresh medium.

\section{Immunoprecipitation and Western blotting}

293 T cells were transfected with each plasmid using FuGENE 6, according to the manufacturer's recommendations. Forty-eight hours after transfection, the cells were lysed in TNE buffer (10 mM Tris-HCl, pH 7.8, 1\% NP-40, $150 \mathrm{mM} \mathrm{NaCl,} 1 \mathrm{mM}$ EDTA) on ice for 15 min. The supernatants were incubated with antic-Myc (9E10; Santa Cruz) or anti-Flag (M2; Sigma) antibody for $1 \mathrm{~h}$ at $4^{\circ} \mathrm{C}$. After adding Protein 
G-Sepharose 6 Fast Flow (GE Healthcare), the samples were incubated for a further $2 \mathrm{~h}$ at $4^{\circ} \mathrm{C}$ and washed five times with TNE buffer. The immunoprecipitates were separated by sodium dodecyl sulfate polyacrylamide gel electrophoresis (SDS-PAGE) and transferred onto polyvinylidene fluoride (PVDF) membranes (Millipore). After blocking, the filters were incubated with anti-c-Myc or anti-Flag antibodies. Stella proteins and Np95 proteins were detected using the anti-Stella antibody [6] and anti-Np95 antibody (MBL; D289-3), respectively. Horseradish peroxidase (HRP)-conjugated sheep anti-mouse IgG (A9174; Sigma) or HRP-conjugated donkey anti-rabbit IgG (NA9340; GE Helthcare) were used as the secondary antibody. The signal was detected using the ECL Western blotting detection reagent (GE Healthcare).

\section{Yeast two-hybrid screening}

The entire coding region of Stella was fused in-frame to the GAL4 DNA-binding domain of the plasmid pGBKT7 (Clontech). Yeast two-hybrid screening was performed using the Matchmaker Gal4 Two-Hybrid System 3 (Clontech). A mouse germinal vesicle $(\mathrm{GV})$-oocyte cDNA library in pGADT7 was obtained from Dr. M. Matzuk (Baylor College of Medicine, Houston, TX). The bait plasmid pGBKT7-Stella was transformed into the yeast strain Y187 and mated with AH109 pre-transformed with GV-oocyte cDNA. The diploid colonies were plated on nutritionally selective plates 
deficient in adenine, histidine, leucine, and tryptophan (SD-Ade-His-Leu-Trp) to screen for binding partners, according to the manufacturer's protocol. Colonies were picked after 7 days of incubation at $30^{\circ} \mathrm{C}$ and re-grown on SD-Ade-His-Leu-Trp plates containing 5-bromo-4-chloro-3-indolyl- $\alpha$-D-galactopyranoside $(\mathrm{X}$ - $\alpha$-gal) to determine the $\beta$-galactosidase activity. Plasmids containing putative Stella-binding cDNAs were recovered from the yeast cells and electroporated into XL1-Blue cells. The resulting plasmids were sequenced using a genetic analyzer (ABI PRISM 3100; Applied Biosystems). To confirm the protein interaction in yeast, cDNAs encoding the two proteins were co-transformed into the yeast strain AH109, and the growth and color were examined on SD-Ade-His-Leu-Trp plates containing X- $\alpha$-gal.

\section{Immunofluorescence}

Control NIH3T3 cells and Stella-expressing NIH3T3 cells were seeded onto glass coverslips. Cells were fixed with $4 \%$ paraformaldehyde (PFA) in phosphate-buffered saline (PBS), permeabilized with $0.5 \%$ Triton X-100 in PBS for $10 \mathrm{~min}$, and blocked in 5\% normal goat serum (NGS) in PBS for $1 \mathrm{~h}$. Rabbit-anti- DNMT1 (1:50 dilution) and mouse-anti-PCNA (1:50 dilution) antibodies were applied to the coverslips and incubated for $1 \mathrm{~h}$. After three washes with $4 \times$ PBS, the samples were incubated with the following secondary antibodies: Alexa568-conjugated rabbit anti-rabbit-IgG $(\mathrm{H}+\mathrm{L})$ 
antibody (1:200; Invitrogen), and Alexa488-conjugated anti-mouse $\operatorname{IgG}(\mathrm{H}+\mathrm{L})$ antibody

(1:200; Invitrogen). Nuclei were counterstained with 4',6-diamidino-2-phenylindole (DAPI; $0.25 \mu \mathrm{g} / \mathrm{mL}$ ). Immunofluorescence was visualized using an LSM510 confocal laser-scanning microscope (Carl Zeiss). Intracellular puncta of Np95 or DNMT1 colocalizing with PCNA were counted. Mid-to-late S phase cells were identified by their distinctive PCNA staining pattern [8] and PCNA-positive replication foci were defined as foci in which the signal intensity was more than twice that of the nucleoplasm. The percent level of colocalization of Np95 or DNMT1 with PCNA-positive foci within individual cells was calculated for 30 cells.

\section{GST pull-down assay}

The GST-Stella fusion proteins were purified from bacterial lysates using glutathione Sepharose 4B (GE Healthcare). The GST-fusion proteins bound to Sepharose beads were incubated with 35S-Met-labeled Np95 proteins in TNE buffer for $3 \mathrm{~h}$ at $4^{\circ} \mathrm{C}$ and washed five times with TNE buffer. The GST precipitates were separated by SDS-PAGE. The gels were exposed on an imaging plate and visualized using the BAS-2000 imaging system (FujiFilm).

\section{Global DNA methylation analysis}


A previously reported protocol for global DNA methylation analysis was used, with slight modifications [9]. Genomic DNA was isolated from various cells with proteinase $\mathrm{K}$ and RNase A, followed by phenol/chloroform extraction and ethanol precipitation. Aliquots of $3 \mu \mathrm{g}$ of genomic DNA were digested with $50 \mathrm{U}$ of methylation-sensitive HpaII or the methylation-insensitive isoschizomer $\mathrm{MspI}$ for $16-18 \mathrm{~h}$ at $37^{\circ} \mathrm{C}$. The digested genomes were purified by phenol/chloroform extraction and ethanol precipitation. Samples of $250 \mathrm{ng}$ of purified DNA were labeled with $3 \mathrm{H}-\mathrm{dCTP}$ at $56^{\circ} \mathrm{C}$ for $1 \mathrm{~h}$, using a single-nucleotide extension reaction. Undigested genomic DNA served as a background control. The samples were applied to DE- 81 ion-exchange filters and washed three times with $0.5 \mathrm{M}$ Na-phosphate buffer $(\mathrm{pH} 7.0)$ at room temperature. The filters were then dried and processed for scintillation counting. The incorporation of ${ }^{3} \mathrm{H}-\mathrm{dCTP}$ into DNA was expressed as mean disintegrations per minute (dpm) per microgram of DNA after subtraction of the dpm incorporation of undigested samples (background). The absolute percent of double-stranded unmethylated CCGG sites was calculated as $\{1-(\mathrm{dpm}$ incorporation after HpaII $) /(\mathrm{dpm}$ incorporation after MspI $)\} \times$ $100 \%$. 


\section{Results and Discussion}

Stella protects the DNA methylation state of the maternal genome, intracisternal A-particle (IAP) retrotransposons, and some imprinted genes in early embryos [10]. To clarify its function in more detail, we overexpressed Stella in NIH3T3 fibroblasts. As shown in Fig. 1A, endogenous Stella was not detected in control NIH3T3 cells, but Stella was detected in the nuclei of Stella-expressing NIH3T3 cells. First, we analyzed the DNA methylation status of the control and Stella expressing NIH-3T3 cells and found that Stella expressing fibroblasts displayed a decreased DNA methylation level (Fig. 1B).

We next examined whether the active or passive demethylation process participates in this DNA hypomethylation by inhibition of the cell cycle. If hypomethylation is caused by a replication-dependent passive process, mitomycin-C-induced cell cycle inhibition would abolish the effect of Stella on DNA hypomethylation. Hypomethylation of DNA by Stella was not observed in the presence of mitomycin $\mathrm{C}$, indicating that the effect was related to passive demethylation.

Yeast two-hybrid analysis of a GV oocyte library using Stella as the bait identified Np95 as a candidate binding partner (Supplementary Table 1). This binding was confirmed by immunoprecipitation (Fig. 2A) and GST pull-down assay (Fig. 2C and D). Analysis of 
various mutants of Np95 fused with GST showed that Stella binds to Np95 through the PHD and SRA domains of Np95. Immunoprecipitation analysis using truncated versions of Stella, Stella $\Delta \mathrm{C}$ (containing amino acids $1-75$ ) and Stella $\Delta \mathrm{N}$ (containing amino acids 76-150), demonstrated that Stella binds to Np95 via its C-terminal region (Fig. 2D).

Because Np95 is an essential protein for the maintenance of DNA methylation, the binding data prompted us to assess the effects of Np95 on Stella-induced DNA hypomethylation. Overexpression of Np95 abolished the effects of Stella-induced DNA hypomethylation; however, it had no effect in the control NIH-3T3 cells (Fig. 3A). Neither Stella $\Delta \mathrm{C}$ nor Stella $\Delta \mathrm{N}$ induced DNA hypomethylation (Fig. 3B). Although Stella $\Delta \mathrm{N}$ bound to Np95 as efficiently as full-length length Stella (Fig. 2D), the protein did not induce global DNA hypomethylation. These data indicate that the C-terminus of Stella is required for the inhibitory effect of Stella on the maintenance of DNA methylation (Fig. 3B). To exclude the possibility that Stella overexpression affected the stability of Np95, we analyzed the protein expression levels of Np95 in NIH3T3 cells, EGFP-expressing cells, and Stella-expressing cells. As shown in Fig. 3C, expression of Stella had virtually no effect on the Np95 protein levels. Taken together, $\underline{\text { Stella induced global DNA hypomethylation was caused by the interaction of Np95 }}$ $\underline{\text { rather than destabilization of } \mathrm{Np} 95 \text { protein. }}$ 
To assess the molecular effects of Stella on DNA hypomethylation in more detail, we investigated the nuclear localization of Np95 and DNMT1. In mid-to-late S phase, PCNA localizes to the replication fork, resulting in a punctate immunostaining pattern in NIH3T3 cells. Because Np95 binds to both hemi-methylated DNA and PCNA, and DNMT1 is recruited by NP95 [5], double immunostaining assays were carried out for Np95 and PCNA, and DNMT1 and PCNA. Both Np95 and DNMT1 localized to the replication fork and showed a punctate immunostaining pattern similar to that of PCNA, and almost all Np95 and DNMT1 colocalized with PCNA (Fig. 4A and C). In contrast, the degrees of colocalization of Np95 and DNMT1 with PCNA in Stella-expressing cells were reduced to less than $10 \%$ and $60 \%$, respectively (Fig. 4B, D).

It is notable that the overexpression of $\mathrm{Np} 95$ alone did not alter the colocalization of Np95 and DNMT1 with PCNA significantly, but did counteract the Stella-induced aberrant localization of Np95 and DNMT1 (Fig. 4A-D). These data strongly indicate that binding of Stella to Np95 disrupts the localization of DNMT1, preventing DNMT1 from effectively maintaining DNA methylation levels.

Numerous reports suggest that aberrant DNA methylation is associated with various diseases, including cancer [11], neurodegenerative disorders, and so on [12]. Despite the large amount of research in this field, it is still unclear whether this aberrant 
DNA methylation is a cause or a consequence of disease. We expect that Stella-mediated global DNA hypomethylation in somatic cells will provide new insight into the role of aberrant DNA methylation in various disease states. 


\section{Acknowledgements}

We thank Drs. D. Trono and M. M. Matzuk for providing lentiviral vectors and the GV-oocyte cDNA library. We also thank Ms. N. Asada for assistance, and Ms. A. Mizokami and M. Imaizumi for secretarial assistance. This work was supported in part by grants from the Uehara Memorial Foundation, the Ministry of Education, Science, Sports, Culture, and Technology of Japan, and by JST, CREST.

\section{References}

[1] S. Feng, S.E. Jacobsen, W. Reik, Epigenetic reprogramming in plant and animal development, Science 330 (2010) 622-627.

[2] Z.D. Smith, A. Meissner, DNA methylation: roles in mammalian development, Nat Rev Genet 14 (2013) 204-220.

[3] M. Okano, S. Xie, E. Li, Cloning and characterization of a family of novel mammalian DNA (cytosine-5) methyltransferases, Nat Genet 19 (1998) 219-220.

[4] M. Bostick, J.K. Kim, P.O. Esteve, A. Clark, S. Pradhan, S.E. Jacobsen, UHRF1 plays a role in maintaining DNA methylation in mammalian cells, Science 317 (2007) 1760-1764.

[5] J. Sharif, M. Muto, S. Takebayashi, I. Suetake, A. Iwamatsu, T.A. Endo, J. Shinga, Y. 
Mizutani-Koseki, T. Toyoda, K. Okamura, S. Tajima, K. Mitsuya, M. Okano, H. Koseki, The SRA protein Np95 mediates epigenetic inheritance by recruiting Dnmt1 to methylated DNA, Nature 450 (2007) 908-912.

[6] T. Nakamura, Y.J. Liu, H. Nakashima, H. Umehara, K. Inoue, S. Matoba, M. Tachibana, A. Ogura, Y. Shinkai, T. Nakano, PGC7 binds histone H3K9me2 to protect against conversion of $5 \mathrm{mC}$ to $5 \mathrm{hmC}$ in early embryos, Nature 486 (2012) 415-419.

[7] S. Morita, T. Kojima, T. Kitamura, Plat-E: an efficient and stable system for transient packaging of retroviruses, Gene Ther 7 (2000) 1063-1066.

[8] H. Leonhardt, H.P. Rahn, P. Weinzierl, A. Sporbert, T. Cremer, D. Zink, M.C. Cardoso, Dynamics of DNA replication factories in living cells, J Cell Biol 149 (2000) 271-280.

[9] I. Pogribny, P. Yi, S.J. James, A sensitive new method for rapid detection of abnormal methylation patterns in global DNA and within $\mathrm{CpG}$ islands, Biochem Biophys Res Commun 262 (1999) 624-628.

[10] T. Nakamura, Y. Arai, H. Umehara, M. Masuhara, T. Kimura, H. Taniguchi, T. Sekimoto, M. Ikawa, Y. Yoneda, M. Okabe, S. Tanaka, K. Shiota, T. Nakano, PGC7/Stella protects against DNA demethylation in early embryogenesis, Nat Cell 
Biol 9 (2007) 64-71.

[11] R. Kanwal, S. Gupta, Epigenetic modifications in cancer, Clin Genet 81 (2012) $303-311$.

[12] Y. Bergman, H. Cedar, DNA methylation dynamics in health and disease, Nat Struct Mol Biol 20 (2013) 274-281. 


\section{Figure Legends}

Figure 1. Cell replication-dependent DNA hypomethylation is induced by Stella.

A) Localization of Stella in NIH3T3 cells. Immunofluorescence analysis of control and Stella-expressing NIH3T3 cells. Stella is shown in red and nuclei are counterstained with DAPI (blue). B) Global DNA methylation status of NIH3T3 cells, Stella-expressing NIH3T3 cells, and Stella-expressing NIH3T3 cells with mitomycin C treatment. Genomic DNA samples purified from cells were digested with the methylation-sensitive HpaII or the methylation-insensitive isoschizomer MspI. The digested materials were labeled with ${ }^{3} \mathrm{H}-\mathrm{dCTP}$ and incorporation of ${ }^{3} \mathrm{H}-\mathrm{dCTP}$ into DNA was measured using a liquid scintillator. Data are expressed as means and standard deviation $(\mathrm{n}=3)$. The DNA methylation level was significantly decreased in Stella-expressing NIH3T3 cells, compared to controls $(* P<0.01, t$-test $)$.

Figure 2. Binding domains of Np95 and Stella.

A) Binding of Stella to Np95. Myc-tagged Stella was co-expressed with FLAG-tagged Np95 in 293T cells. Stella and Np95 were immunoprecipitated with anti-myc and anti-FLAG antibodies, respectively. The immunoprecipitates were analyzed by Western blotting. B) Schematic representation of the wild type and Np95 mutants. All constructs 
were tagged with GST. C) GST pull-down assay of Stella and Np95 mutants. The numbers at the top correspond to those shown in B. D) Binding of truncated versions of Stella with Np95. Myc-tagged Stella, Stella $\Delta$ C, or Stella $\Delta N$ was co-expressed with FLAG-tagged Np95 in 293T cells. Stella, or its deletion mutants, and Np95 were immunoprecipitated with anti-FLAG and anti-Myc antibodies, respectively. The immunoprecipitates were analyzed by Western blotting.

\section{Figure 3. Stella-induced DNA hypomethylation is inhibited by Np95 and N and C} terminal deletion mutants fail to induce DNA hypomethylation.

A) The global DNA methylation status of NIH3T3 cells, Stella-expressing NIH3T3 cells, Np95-expressing NIH3T3 cells, and NIH3T3 cells co-expressing Stella and Np95. Data are expressed as means and standard deviation $(n=3)$. The DNA methylation level was significantly decreased in Stella-expressing NIH3T3 cells, compared to controls $\left({ }^{*} P<0.01, t\right.$-test). B) The global DNA methylation status of control EGFP, Stella, Stella $\Delta C$, and Stella $\Delta$ N-expressing NIH3T3 cells was analyzed. Data are expressed as means and standard deviation $(\mathrm{n}=3)$. The DNA methylation level was significantly decreased in full length (FL) Stella-expressing NIH3T3 cells compared to controls $\left({ }^{*} P\right.$ $<0.01, t$-test). C) Expression levels of Stella and Np95 proteins in NIH3T3 cells, 
EGFP-expressing cells, and Stella-expressing cells. $\beta$-actin was used as a loading $\underline{\text { control. }}$

\section{Figure 4. Stella inhibits the co-localization of Np95 and DNMT1 with PCNA.}

A) Immunofluorescence analysis of Np95 and PCNA in NIH3T3 cells, Stella-expressing NIH3T3 cells, Np95-expressing NIH-3T3 cells, and NIH3T3 cells co-expressing Stella and Np95. Np95 and PCNA are shown in red and green, respectively; nuclei were counterstained with DAPI (white). B) Percentage colocalization of Np95 with PCNA in NIH3T3 cells, Stella-expressing NIH3T3 cells, Np95-expressing NIH-3T3 cells, and NIH3T3 cells co-expressing Stella and Np95. Data are expressed as means and standard deviations $(\mathrm{n}=30)$. The colocalization of Np95 and PCNA was significantly decreased in Stella-expressing NIH3T3 cells, compared to controls $(* P<0.001, t$-test $)$. Similar results were obtained in at least two independent experiments and representative results are shown. C) Immunofluorescence analysis of DNMT1 and PCNA in NIH3T3 cells, Stella-expressing NIH3T3 cells, Np95-expressing NIH-3T3 cells, and NIH3T3 cells co-expressing Stella and Np95. DNMT1 and PCNA are shown in red and green, respectively; nuclei were counterstained with DAPI (white). D) Percentage colocalization of DNMT1 with PCNA 
in NIH3T3 cells, Stella-expressing NIH3T3 cells, Np95-expressing NIH-3T3 cells, and Stella and Np95 co-expressing NIH-3T3 cells. Data are expressed as means and standard deviations $(\mathrm{n}=30)$. The colocalization of Dnmt1 and PCNA was significantly decreased in Stella-expressing NIH3T3 cells, compared to controls $(* P<0.001, t$-test). $\underline{\text { Similar results were obtained in at least two independent experiments and representative }}$ $\underline{\text { results are shown. }}$ 
Figure 1

A

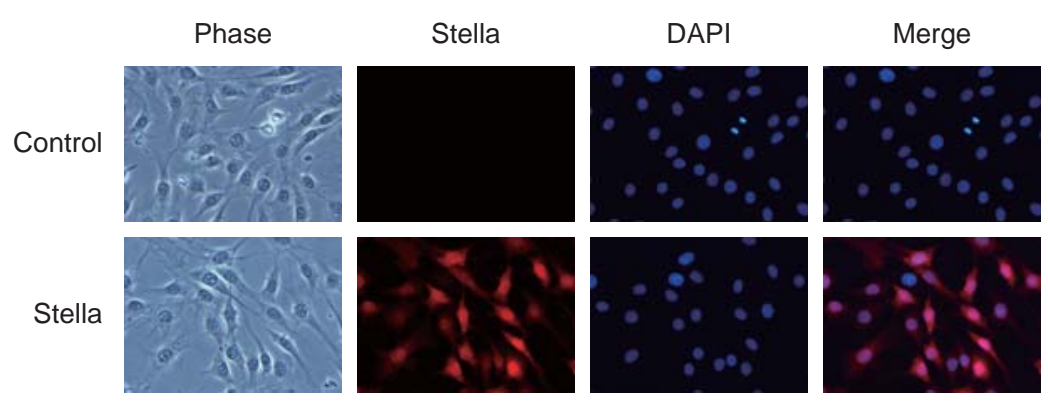

B

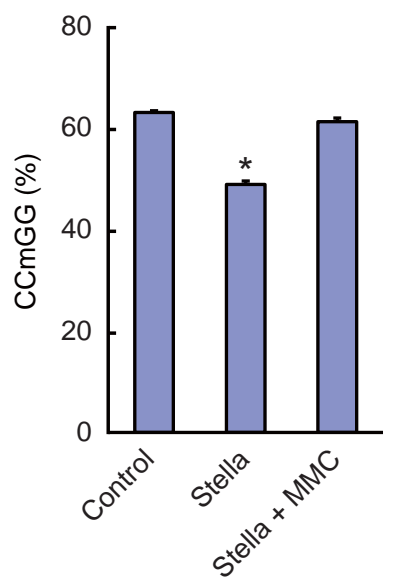


Figure 2

A

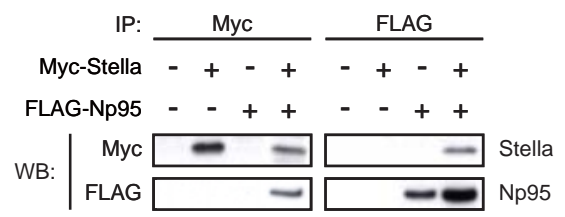

B

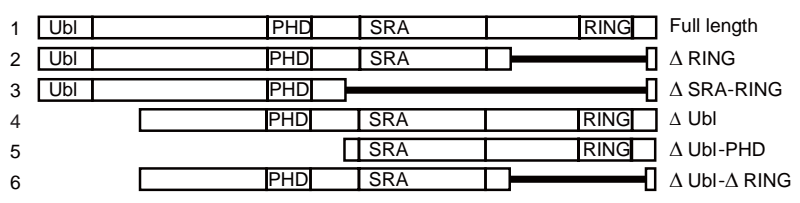

C

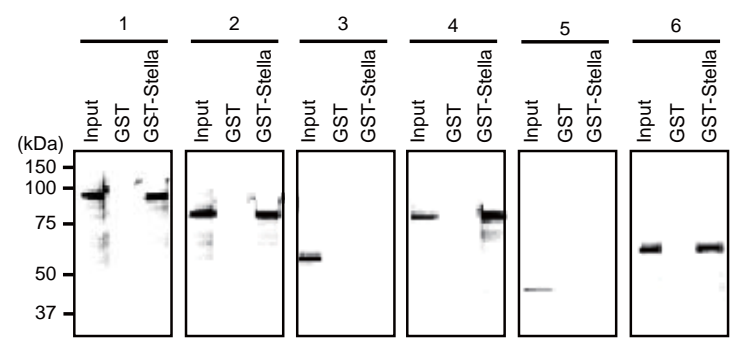

D

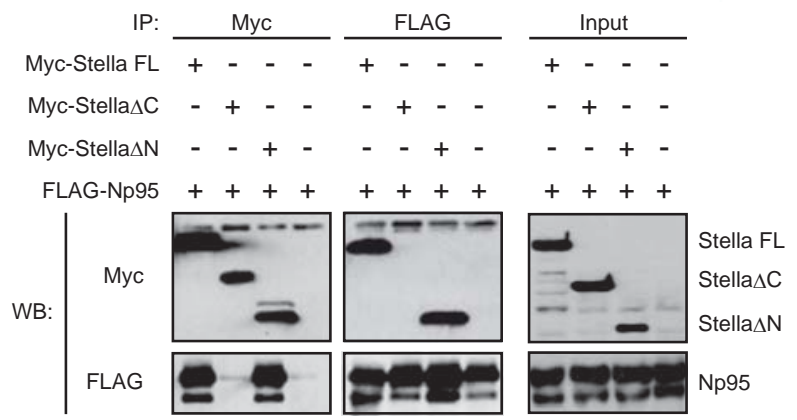


Figure 3

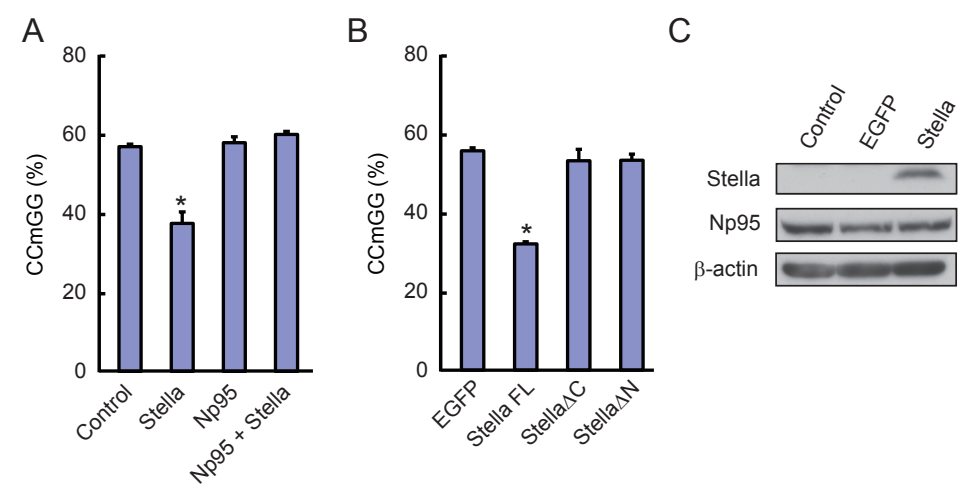


Figure 4

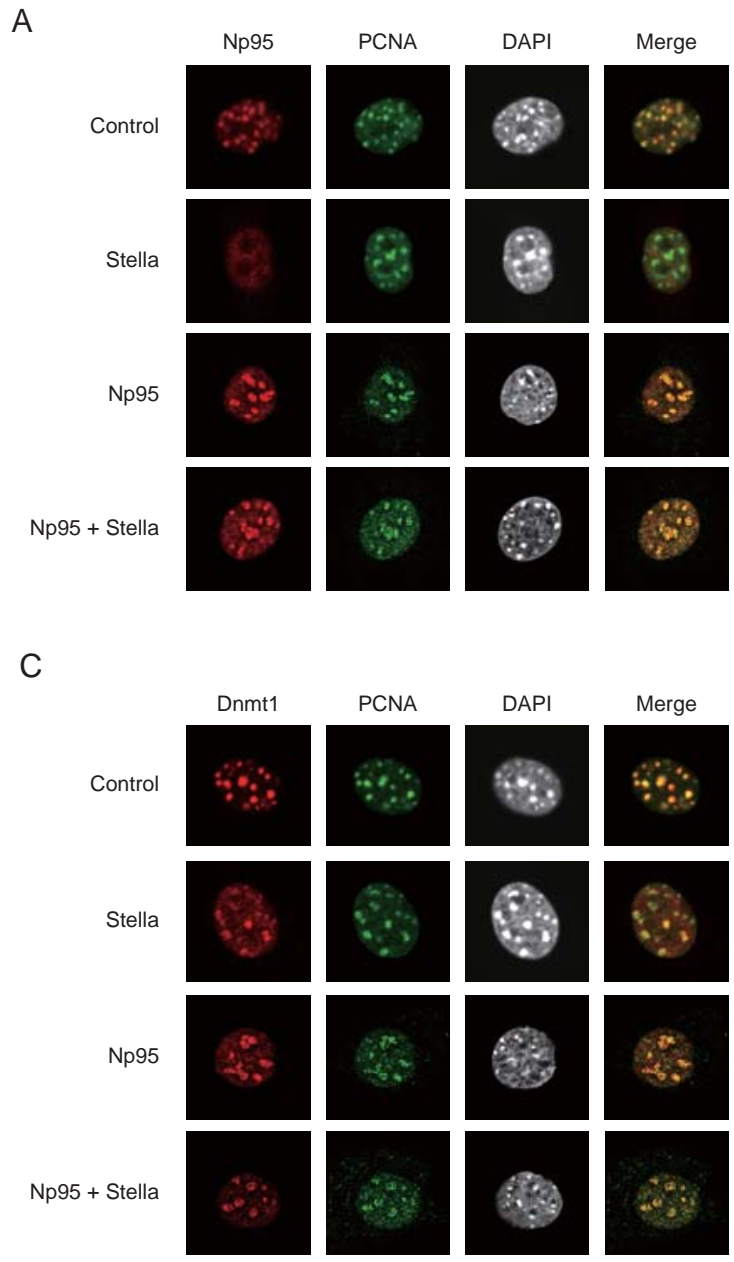

B

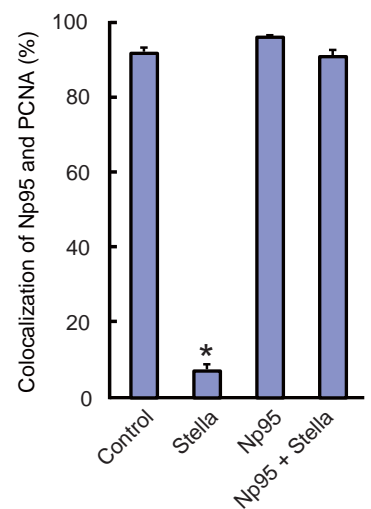

D

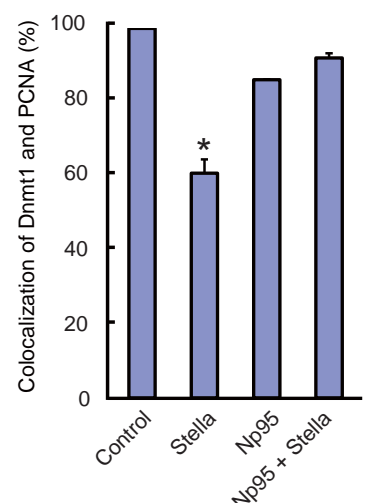

